\title{
Efficient Adaptive Routing in Delay Tolerant Networks
}

\author{
Cong Liu and Jie Wu \\ Department of Computer Science and Engineering \\ Florida Atlantic University \\ Boca Raton, FL 33431
}

\begin{abstract}
Conventional routing algorithms in mobile ad hoc networks (MANETs), i.e., multi-hop forwarding, assume the existence of contemporaneous source-destination paths and are not scalable to large networks. On the other hand, in delay tolerant networks (DTNs), routing protocols use the mobilityassisted, store-carry-forward paradigm which allows delivery among disconnected network components. Adaptive routing, which combines multi-hop and mobility-assisted routing protocols, is of practical value: it allows efficient multi-hop forwarding while providing the flexibility to deliver messages among disconnected network components. However, existing adaptive routing protocols use mobility-assisted routing protocols as an alternative only when the former fails. In this paper, we propose to improve the performance of adaptive routing from a resource allocation point of view, in situations where bandwidth is a critical and limited resource affecting routing performance. We propose an adaptive routing protocol, named efficient adaptive routing (EAR), which allocates bandwidth (or forwarding opportunities) between its multi-hop forwarding component and its mobility-assisted routing component dynamically to improve bandwidth utility. Simulations are conducted to evaluate the routing performance of EAR under different network parameters.

Index Terms-Adaptive routing, delay tolerant networks (DTNs), mobile ad hoc networks (MANETs), simulation.
\end{abstract}

\section{INTRODUCTION}

In conventional mobile ad hoc networks (MANETs), routing algorithms [1], [2] assume that contemporaneous sourcedestination paths always exist and messages are delivered in a single-copy, multi-hop manner. On the other extreme, delay tolerant network (DTN) routing algorithms [3], [4], [5], [6], [7], [8], assume that the network is very sparse and highly mobile, and messages are delivered in a multi-copy, mobilityassisted manner.

Existing multi-hop routing protocols in MANETs and mobility-assisted routing protocols in DTNs can be combined to increase adaptivity in unforeseen network scenarios. In this paper, we focus on adaptive routing, which is able to use multi-hop forwarding (with proactive route maintenance or reactive route discovery) and mobility-assisted forwarding in an addictional manner. The challenge lies in coordinating these two routing components efficiently to improve routing performance in terms of delivery rate.

Previous works have used adaptive routing to fill the gap between multi-hop routing and mobility-assisted routing algorithms. Mirco et al. [9] propose to use Destination-Sequenced Distance-Vector (DSDV) [2] for routing in the same connected network component and then focus on a mathematical framework to calculate the utility of each mobility-assisted forwarding when the destination is not in the same component. Ott et al. [10] propose an integrated multi-hop and mobility-assisted protocol, where a modified Ad hoc On-Demand Distance Vector Routing (AODV) [1] is proposed which broadcasts routing requests that search for the destination and the available DTNenabled nodes at the same time. When AODV fails, mobilityassisted routing is used as an alternative.

All existing adaptive routing algorithms always prioitize the multi-hop routing and use the mobility-assisted routing as a backup to enhance reachability. Such routing protocols could be less efficient than mobility-assisted routing protocols, for example, in networks with high nodal mobility or high traffic rates. This paper focuses on the coordination of the two forwarding protocols in adaptive routing and investigates bandwidth allocation between them, which has not been studied before. We propose efficient adaptive routing (EAR), which contains a simple multi-hop routing component and a simple mobility-assisted routing component. The objective of EAR is to improve bandwidth utility by dynamically allocating bandwidth to these two routing components according to realtime statistics in DTNs when network parameters, such as network density and nodal mobility patterns, are unknown.

The contributions of this paper are summarized as follows.

1) We show the possibility of improving the routing performance of the adaptive routing protocol from a resource allocation perspective.

2) We propose a heuristics to allocate bandwidth between the two components in our proposed protocol EAR.

3) We perform simulation to evaluate the adaptive performance of EAR under a wide range of network parameters.

This paper is organized as follows. Section II presents the basic idea of EAR. Section III describes our method on bandwidth allocation. Section IV shows our simulation methods and results. Finally, Section V concludes the paper.

\section{EFFicient AdAptive Routing (EAR)}

The EAR routing protocol allocates bandwidth between its two routing components by limiting the maximum bandwidth consumed by the multi-hop forwarding component. We define a logical cloud for each node, and we limit bandwidth con- 


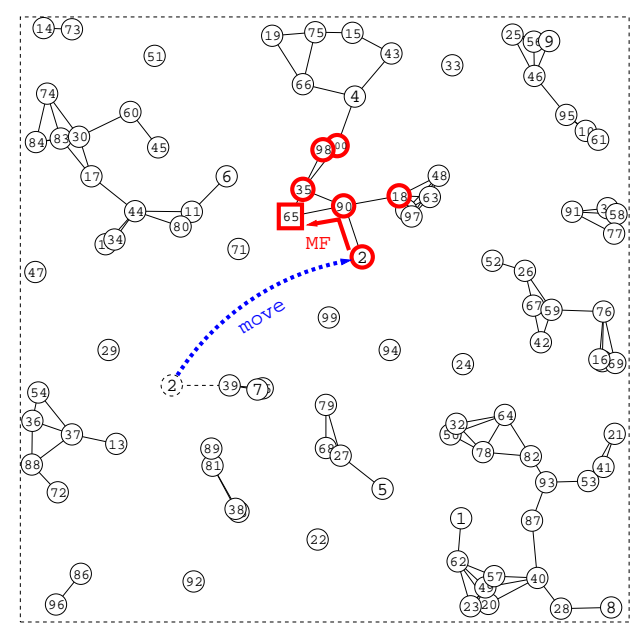

Fig. 1. A message is received by node 65 from node 2 (whose logical cloud includes node 65) through a multi-hop forwarding (MF).

sumption of the multi-hop forwarding by restricting a node to only forward messages to other nodes in its logical cloud.

Definition 1 (Logical cloud): The logical cloud $S$ of a node $u$ is a set of nodes such that, for any $v \in S$, there exists a path from $u$ to $v$ consisting of other nodes in $S$. The minimum logical cloud contains $u$ and its 1-hop neighbors.

According to Definition 1, the logical cloud of node $u$ is a subset of the physical connected network component containing $u$. We limit the number of nodes in the logical cloud of $u$ as follows. Given a constant $C$ for logical cloud size, (1) add $u$ and its 1-hop neighbors to the logical cloud, (2) while the size of the logical cloud is smaller than $C$, add to it one of $u$ 's $k$-hop neighbors $v$ that has the highest priority $p_{r}=\left(\frac{1}{k}, v\right)$ among nodes that are not in the logical cloud.

In the priority $p_{r}$, a smaller hop-count is the first priority and ties are broken by comparing IDs. As an example, in Figure 1, the logical cloud of node 65 with $C=7$ originally consists of nodes $18,35,65,90,98,100$, and 97. After node 2 connects to node 90 , the local cloud was updated by replacing node 97 with node 2 .

With logical cloud, the bandwidth consumed by the multihop forwarding protocol of a node is independent of the network size. Logical cloud is also used to limit the bandwidth consumption in proactive/reactive shortest paths maintenance.

For simplicity, in EAR, we use DSDV [2] as the multi-hop routing component, and spray-and-wait [11] as the mobilityassisted routing component. In future work, they can be replaced by other protocols, such as AODV [1] and sprayand-focus [12].

\section{A. The DSDV multi-hop routing component}

DSDV [2] is a table-driven routing scheme for ad hoc mobile networks based on the Bellman-Ford algorithm. In DSDV, each node maintains a hop-count of the shortest path to every other node. The DSDV routing component differs from the original DSDV in that each node only maintains the hop-count of the shortest path to the nodes in its logical cloud.
Let $C$ be the average logical cloud size. The amortized bandwidth consumption of the proactive route maintenance and the average size of the routing tables is $O(C)$.

\section{B. Spray-and-wait mobility-assisted routing component}

The mobility-assisted routing component used in EAR is spray-and-wait [11]. In the original spray-and-wait, each new message is first forwarded to a fixed number, $L$, of nodes, and one of these $L$ nodes will deliver the message when it encounters the destination.

A variation of spray-and-wait is used in EAR, which differs from spray-and-wait in that there is no maximum number $L$, which means spray-and-wait can use the rest of the bandwidth to maximize the delivery probabilities of the messages. Specifically, each message is associated with a logical ticket whose value is initially 1.0. Whenever a message is copied to another node, the tickets' values on the sender and the receiver equal half of the original ticket value. To ensure that all messages have equal chances of being sprayed to other nodes, a node first sprays the messages with larger ticket values.

\section{A Heuristic FOR EFFicient BANDWidTh ALLOCATION}

It is important to find an appropriate bandwidth allocation to improve the overall routing performance of the adaptive routing protocols. We present a heuristic for efficient bandwidth allocation by maximizing bandwidth utility, assuming that the network is homogeneous (in which each node forwards messages for other nodes) and the nodes' mobility is randomized (such as in a random waypoint mobility model).

Our method for allocating bandwidth is to determine the logical size $C$ for all nodes. After $C$ is determined, each node selects nodes in its logical cloud. Whenever a node has a forwarding opportunity, it first forwards messages whose destinations are in its logical cloud (closer destinations first), and then sprays messages whose destinations are not in the logical cloud.

We define $B$ to be the available total bandwidth of a node. In other words, $B$ is the volume of all messages that can be received or sent per node per unit of time. For adaptive routing, $B$ includes the bandwidth consumed by data messages, the routing information of mobility-assisted routing (which is 0 for spray-and-wait), and the proactive route maintenance messages for the multi-hop forwarding protocol. Let $R$ be the average volume of data messages delivered per destination per unit of time. Obviously, $R \leq B$.

Definition 2 (Bandwidth utility): The bandwidth utility $U$ of a routing protocol is the ratio of the volume of the data messages delivered to the volume of total messages consumed in the network. $U=R / B$.

The objective of the efficient bandwidth allocation is to achieve the highest bandwidth utility $U$ by properly dividing the total bandwidth $B$ into three parts (1) $B_{S}$, the bandwidth allocated to spraying copies of messages in the network, (2) $B_{F}$, the bandwidth allocated to the multi-hop forwarding, 
and (3) $B_{P}$, the bandwidth allocated to the proactive route maintenance in the logical cloud of each node. Here, $B_{F}$ and $B_{P}$ are implicitly related. We will approximate $B_{S}, B_{F}$, and $B_{P}$ respectively as functions of the average cloud size $C$. Then, we use a heuristic to find a $C$ under which an efficient bandwidth allocation is achieved.

\section{A. The bandwidth consumption of spraying}

Suppose logical cloud size $C$ is 1 , i.e., the logical cloud of each node $u$ contains no other node but $u$ itself and none of the nodes know the identity of their neighbors. In such a scenario, a number of copies of each message are sprayed into the network, but no copy can be further forwarded to its destination. The volume of data messages received per node per time unit $R_{1}=B_{S} / N$ (1 denotes that the size of the logical cloud is 1), where $B_{S}$ is the amount of bandwidth consumed by spraying copies of messages and $N$ is the number of nodes in the network. $R_{1}=B_{S} / N$ because the probability of the destination of the message being $u$ is $\frac{1}{N}$ when a node $u$ receives a message.

\section{B. The bandwidth consumption of forwarding}

We assume that messages received by each node in the spraying scheme are independent, i.e., two nodes will not have more common messages because they are geometrically closer to each other. This assumption is acceptable when the nodes' mobility is high enough. If the logical cloud size is $C>1$, then the volume of messages received per node per time unit $R_{C}=R_{1} C$. This is because for each node $u$ the rate at which it receives messages whose destination is $u$ is $R_{1}$, and so is the rate of any other nodes receiving messages with destination $u$. If all $C$ nodes in $u$ 's logical cloud can successfully forward to $u$ the messages they received from spraying whose destinations is $u$, the receiving rate of $u$ is increased by $C$ times. Therefore, with logical cloud size $C$, the receiving rate $R_{C}=R_{1} C=\frac{B_{S} C}{N}$.

Let $B_{F}$ be the bandwidth consumed by forwarding messages inside logical clouds, $K$ be the average hop-count between a node and another node in its logical cloud, then $B_{F}=R_{C} K$. This is because, in a homogeneous network, a node having a receiving rate $R_{C}$ suggests that it has the same receiving rate $R_{C}$ of messages that are destined to any other nodes in its logical cloud. Therefore, the bandwidth $B_{F}$ consumed by forwarding these messages to their destinations in the same cloud is $R_{C} K$. We approximate $K$ by $C / 2$ considering that, in sparse networks, connected components are likely to have a linear topology (as can be observed in Figure 1). Then, we have,

$$
B_{F}=R_{C} K=R_{C} \frac{C}{2}=\frac{B_{S} C}{N} \frac{C}{2}=\frac{B_{S} C^{2}}{2 N} .
$$

C. The bandwidth consumption of proactive route maintenance

If the average logical cloud size is $C$, the average bandwidth consumed in the maintenance of the shortest paths of a node in its logical cloud is $O(C)$. This is because each node needs to periodically broadcast its routing table containing items for each node in its logical cloud, whose size is $C$. Let the bandwidth consumed by the proactive maintenance be $B_{P}$, then $B_{P}=M C$, where $M$ is a constant that depends on the frequency of the periodical broadcast and the size of data item for each node in the logical cloud.

\section{Maximum bandwidth utility}

We use Theorem 1 to approximate the maximum bandwidth utility.

Theorem 1: The maximum bandwidth utility is approximately achieved when the average logical cloud size $C$ is $\sqrt{2 N}$, where $N$ is the network size.

Proof: The average bandwidth utility can be approximated by,

$$
U=\frac{R_{C}}{B}=\frac{R_{C}}{B_{S}+B_{F}+B_{P}}=\frac{\frac{B_{S} C}{N}}{B_{S}+\frac{B_{S} C^{2}}{2 N}+M C} .
$$

Assuming $B_{S}$ is independent of $C$, to maximize the bandwidth utility $U$, we let $\frac{d U}{d C}=0$.

$$
\begin{gathered}
\frac{d U}{d C}=\frac{d\left(\frac{\frac{B_{S} C}{N}}{B_{S}+\frac{B_{S} C^{2}}{2 N}+M C}\right)}{d C} \\
=\frac{\left(B_{S}+\frac{B_{S} C^{2}}{2 N}+M C\right) d\left(\frac{B_{S} C}{N}\right)-\frac{B_{S} C}{N} d\left(B_{S}+\frac{B_{S} C^{2}}{2 N}+M C\right)}{\left(B_{S}+\frac{B_{S} C^{2}}{2 N}+M C\right)^{2}} \\
=\frac{\left(B_{S}+\frac{B_{S} C^{2}}{2 N}+M C\right) \frac{B_{S}}{N}-\frac{B_{S} C}{N}\left(\frac{B_{S} C}{N}+M\right)}{\left(B_{S}+\frac{B_{S} C^{2}}{2 N}+M C\right)^{2}} .
\end{gathered}
$$

When $\frac{d U}{d C}=0$, we have,

$$
\begin{gathered}
\left(B_{S}+\frac{B_{S} C^{2}}{2 N}+M C\right) \frac{B_{S}}{N}-\frac{B_{S} C}{N}\left(\frac{B_{S} C}{N}+M\right)=0 \\
\Rightarrow\left(B_{S}+\frac{B_{S} C^{2}}{2 N}+M C\right)-C\left(\frac{B_{S} C}{N}+M\right)=0 \\
\Rightarrow B_{S}+\frac{B_{S} C^{2}}{2 N}+M C-\frac{B_{S} C^{2}}{N}-M C=0 \\
\Rightarrow B_{S}-\frac{B_{S} C^{2}}{2 N}=0 \Rightarrow C=\sqrt{2 N} .
\end{gathered}
$$

Theorem 1 shows that the maximum bandwidth utility can be achieved approximately by selecting an average cloud size $C=\sqrt{2 N}$ where $N$ is the network size. If the network size $N$ is unknown, $C$ can be approximated from $B_{S}$ and $R_{C}$, which can be estimated statistically by nodes based on message transmission history. Since,

$$
R_{C}=\frac{B_{S} C}{N} \Rightarrow N=\frac{B_{S} C}{R_{C}},
$$

we have,

$$
C^{2}=2 N=2 \frac{B_{S} C}{R_{C}} \Rightarrow C=\frac{2 B_{S}}{R_{C}} .
$$


TABLE I

SiMULATION PARAMETERS

\begin{tabular}{|l|l|l|}
\hline Parameters & Default & Range \\
\hline Field size & $1,000 \times 1,000\left(\mathrm{~m}^{2}\right)$ & \\
Number of nodes & 200 & $50-250$ \\
Message rate & $1(\mathrm{msgs} / \mathrm{s})$ & $1-10(\mathrm{msgs} / \mathrm{s})$ \\
Buffer size & 1,000 & $100-1,000$ \\
Data message size & $2 \mathrm{~KB}$ & \\
Radio bandwidth & $1 \mathrm{Mb}$ & \\
Transmission range & $100(\mathrm{~m})$ & $100-1,000(\mathrm{~s})$ \\
Message TTL & $1,000(\mathrm{~s})$ & \\
Simulation time & $2,000(\mathrm{~s})$ & $20-200(\mathrm{~s})$ \\
Moving speed in RWP & $100(\mathrm{~m} / \mathrm{s})$ & \\
Pause time in RWP & $50(\mathrm{~s})$ & \\
\hline
\end{tabular}

An algorithm to maximize bandwidth utility is described as follows: (1) $C=1$ initially, (2) $C$ is updated periodically according to the current statistics of $B_{S}$ and $R_{C}: C$ is increased by 1 if $C<\frac{2 B_{S}}{R_{C}}$, and (3) $C$ is decreased by 1 if $C>\frac{2 B_{S}}{R_{C}}$.

\section{Simulation \& Results}

\section{A. Implementation \& settings}

We implemented our simulation on our EASIM simulator [13] which extends the JiST/SWANS simulator [14]. Our implementation of a DTN node includes (1) a neighbor discovery mechanism using periodical beacons, (2) a reliable broadcast operation using delayed acknowledgments, (3) a message vector exchange mechanism which reduces redundant maintenance message forwarding, (4) a buffer management mechanism, (5) DSDV, and (6) spray-and-wait.

To draw a comparison, we also implemented two other protocols which can be regarded as variations of EAR: the spray-and-wait protocol [11] whose logical cloud is limited to 1-hop neighbors, and an adaptive DTN routing protocol which has no limitation on the size of its logical cloud, which is simply denoted as adaptive. Our metrics are bandwidth utility and delivery rate. Simulation parameters are network size, nodal mobility step, message time-to-live (TTL), and message buffer size. The main simulation parameters are summarized in Table I.

\section{B. Simulation results and discussions}

In the first set of simulations (Figures 2(a) and 2(b)), we vary the number of nodes from 50 to 250 . As shown in Figure 2(a), the bandwidth utility of EAR and spray-and-wait increases almost linearly as the number of nodes increases. This is because as density increases, each broadcast can send a message to more nodes. The bandwidth utility of sprayand-wait is, on average, $10 \%$ smaller than EAR and EAR is the best under different numbers of nodes. This shows that EAR does in fact adaptively improve the bandwidth utility. The bandwidth utility of adaptive stops increasing as the number of nodes is more than 200. This is because the size of the connected component increases as the number of nodes increases. The bandwidth used by multi-hop forwarding increases and eventually consumes all the bandwidth.

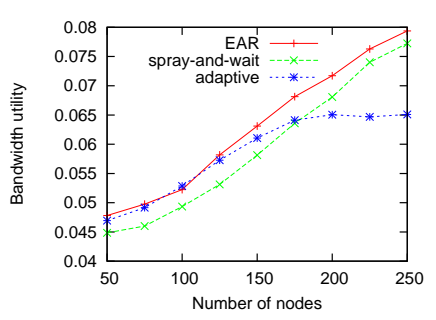

(a) Bandwidth utility

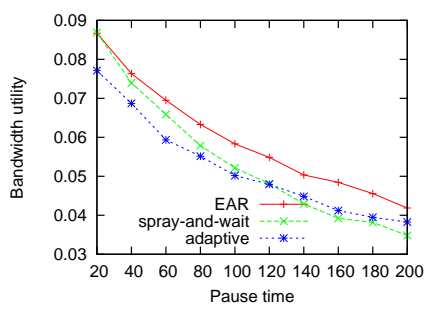

(c) Bandwidth utility

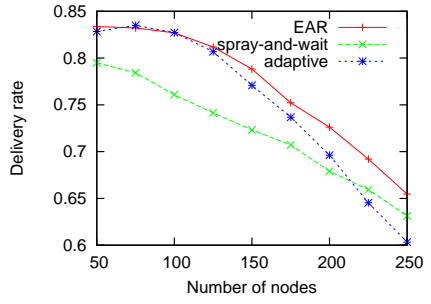

(b) Delivery rate

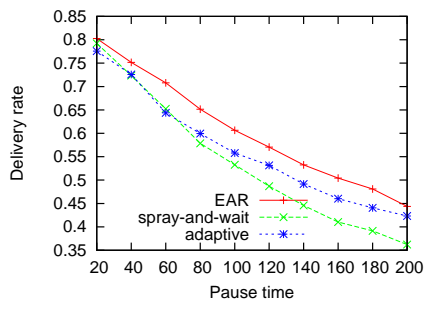

(d) Delivery rate
Fig. 2. Performance comparison with different message pause time and number of nodes.

Figure 2(b) shows that the delivery rate of all routing algorithms decreases as the number of nodes increases. The delivery ratio of EAR is $5-15 \%$ better than that of spray-andwait. That of adaptive shows the worst degradation among all protocols as the number of nodes increases, and its performance becomes the worst when the number of nodes is over 200. Looking at Figure 2(a), we can see that the delivery rates are closely related to the bandwidth utility - the protocols that have better bandwidth utility also have better delivery ratios. This can be explained by the definition of bandwidth utility.

In the second set of simulations (Figures 2(c) and 2(d)), we increase the nodes' pause time in the random waypoint mobility model from 20 to 200. As shown in Figure 2(c), the bandwidth utility of all protocols decreases as pause time increases. This is because the spray-and-wait component relies on the mobility of the network. Moreover, when mobility is extremely low, a message is not guaranteed to be delivered within its TTL, even with infinite bandwidth. The bandwidth utility of EAR is $10-30 \%$ higher than that of adaptive and is also higher than that of spray-and-wait by up to $20 \%$.

Figure 2(d) shows that the delivery rate of all protocols decreases as pause time increases, and the trends are much the same as the bandwidth utility shown in Figure 2(c). EAR has the highest delivery rate and when the pause time is 200 seconds, the delivery rate of EAR is $50 \%$ higher than that of spray-and-wait.

In the third set of simulations (Figures 3(a) and 3(b)), we vary the message TTL from 100 to 1,000 seconds. As shown in Figure 3(a), the bandwidth utilities of all protocols increase as the messages' TTL increases. The bandwidth utility of EAR is the best under different message TTLs. The bandwidth utility of spray-and-wait does not increase as significantly as the other two protocols. When message TTL is larger than 500 


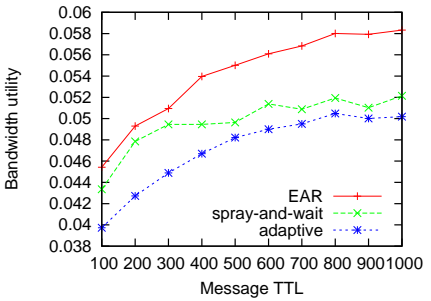

(a) Bandwidth utility

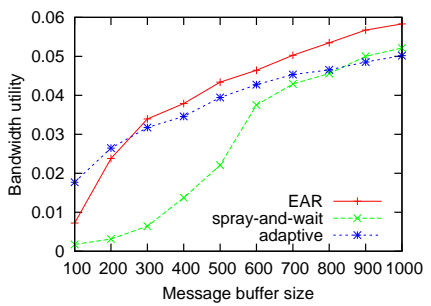

(c) Bandwidth utility
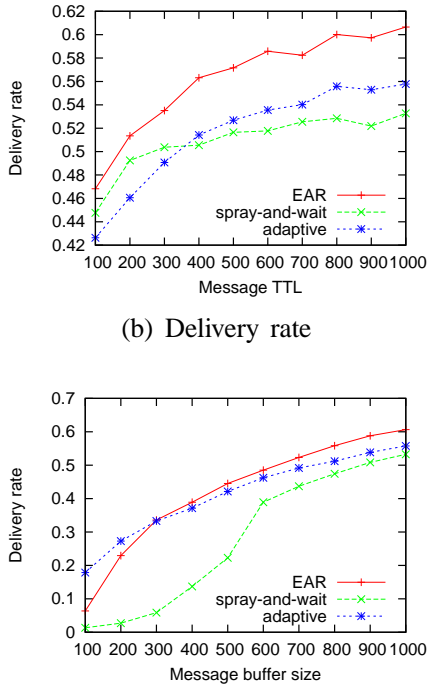

(d) Delivery rate (b) Delivery rate

Fig. 3. Performance comparison with different TTLs and message buffer sizes.

seconds, the bandwidth utility of EAR is at least $15 \%$ higher than those of spray-and-wait and adaptive.

Figure 3(b) shows that the delivery rates of all protocols increase as message TTL increases, and EAR has the highest delivery rate under different message TTLs. When message TTL is greater than 500 seconds, the improvement in delivery rate when using EAR as opposed to spray-and-wait and adaptive are $10 \%$ and $15 \%$ respectively.

In the last set of simulations (Figures 3(c) and 3(d)), we vary the message buffer size from 100 to 1,000 messages per node. As shown in Figure 3(c), as the message buffer size increases, the bandwidth utility increases for all protocols. This is because as the buffer size decreases, some of the messages have to be removed before they expire. From the figure, adaptive has the best performance with a very small buffer. The bandwidth utility of spray-and-wait is the most sensitive to buffer size, and it degrades most significantly when the buffer size is smaller than 600 messages. Comparatively, EAR is more tolerant to a small buffer size: its bandwidth utility is worse than adaptive's only when the buffer size is smaller than 300 messages.

Figure 3(d) shows that the protocols' delivery rates change in the same way as bandwidth utility when the message buffer size changes. Figure 3(d) shows that EAR and adaptive are much better than spray-and-wait as the message buffer is small.

\section{Summary of simulation}

The simulation results show that EAR outperforms other protocols in terms of delivery rate since it has a better bandwidth utility. Thus, it can be concluded that the delivery rate is closely related to the bandwidth utility. From the simulation results, we found that the performance of EAR does not decrease when the performance of one of the other two deteriorates and the other does not. This shows that EAR combines the advantages of the two protocols, and it can improve the routing performance with its ability to allocate more bandwidth to the better routing component under different network settings.

\section{CONCLUSiOns}

In this paper, we proposed to improve the performance of adaptive routing from a resource allocation point of view, called efficient adaptive routing (EAR), in situations where bandwidth is a critical and limited resource affecting routing performance. Simulation results show our proposed routing protocol, EAR, has better routing performance than the compared protocols. We allocate bandwidth between proactive routing and spray-and-wait by limiting the logical cloud size. Future research may consider more complicated proactive/reactive routing components and other mobility-assisted routing component, and use other strategies to allocate bandwidth.

\section{ACKNOWLEDGMENT}

This work was supported in part by NSF grants CNS 0422762, CNS 0434533, CNS 0531410, CCF 0545488, and CNS 0626240. Email: jie@cse.fau.edu.

\section{REFERENCES}

[1] S. R. Das, C. E. Perkins, and E. M. Royer. Performance comparison of two on-demand routing protocols for ad hoc networks. In Proc. of IEEE INFOCOM, 2002.

[2] C. Perkins and P. Bhagwat. Highly dynamic destination-sequenced distance-vector routing (DSDV) for mobile computers. In Proc. of Conference on Communications Architectures, Protocols and Applications in conjection with SIGCOMM 94, 1994.

[3] J. Ghosh, S. J. Philip, and C. Qiao. Sociological orbit aware location approximation and routing (SOLAR) in MANET. In Proc. of ACM MobiHoc, 2005.

[4] J. Haas, J. Y. Halpern, and L. Li. Gossip-based ad hoc routing. In Proc. of IEEE INFOCOM, 2002.

[5] J. Leguay, T. Friedman, and V. Conan. DTN routing in a mobility pattern space. In Proc. of ACM SIGCOMM Workshop on Delay-Tolerant Networking, 2005.

[6] C. Liu and J. Wu. Routing in a cyclic mobispace. In Proc. of ACM MobiHoc, 2008.

[7] S. Merugu, M. Ammar, and E. Zegura. Routing in space and time in network with predictable mobility. In Technical Report: GIT-CC-04-07, College of Computing, Georgia Tech, 2004.

[8] A. Vahdate and D. Becker. Epidemic routing for partially-connected ad hoc networks. In Technical Report, Duke University, 2002.

[9] M. Mirco, H. Stephen, and M. Cecilia. Adaptive Routing for Intermittently Connected Mobile Ad Hoc Networks. In Proc. of IEEE WoWMoM, June 2005.

[10] J. Ott, D. Kutscher, and C. Dwertmann. Integrating DTN and MANET routing. In Proc. of ACM SIGCOMM Workshop on Challenged Networks (CHANTS), 2006.

[11] T. Spyropoulos, K. Psounis, and C. Raghavendra. Spray and Wait: An Efficient Routing Scheme for Intermittently Connected Mobile Networks. In Proc. of ACM WDTN, 2005.

[12] T. Spyropoulos, K. Psounis, and C. Raghavendra. Spray and focus: Efficient mobility-assisted routing for heterogeneous and correlated mobility. In Proc. of the International Workshop on Intermittently Connected Mobile Ad hoc Networks (ICMAN), in conjunction with IEEE PerCom, 2007.

[13] C. Liu. EASIM ad hoc network simulator. http://www.geocities.com/gzcliu_proj/.

[14] http://jist.ece.cornell.edu/. 